\title{
Endovascular treatment of vein of Galen aneurysmal malformation
}

\author{
J du Toit, $M B C h B$ \\ J A Bam, $M B C h B$ \\ V Mngomezulu, MB BCh, FCRad (D) (SA), MBA \\ Department of Radiology, Dr George Mukhari Hospital, University of Limpopo \\ (MEDUNSA Campus), Ga-Rankuwa
}

\section{Introduction}

Vein of Galen aneurysmal malformation (VGAM) accounts for up to $30 \%$ of paediatric vascular malformations. ${ }^{1}$ As the internal cerebral veins develop, the anterior portion of the median prosencephalic vein of Markowski (MPM) regresses, and the posterior portion remains to form the vein of Galen. It is believed that the VGAM is the result of an arteriovenous connection between the primitive choroidal arteries and the MPM. ${ }^{2}$ The abnormal flow in these connections inhibits the involution of the MPM. ${ }^{3}$

\section{Case report}

An 11-month-old boy presented with a progressively increasing head circumference. He had normal milestone development up to the date of admission, with no cardiac abnormalities.

The patient underwent computed tomography (CT) scan of the head, which showed obstructive hydrocephalus and an aneurysmally dilated vein of Galen, a stenotic straight sinus, and a distended torcular Herophili. Prominence of the sulci and gyri were also evident (Fig.1a). These findings were confirmed on magnetic resonance imaging (MRI), angiography (MRA) and magnetic resonance venography (MRV) (Figs

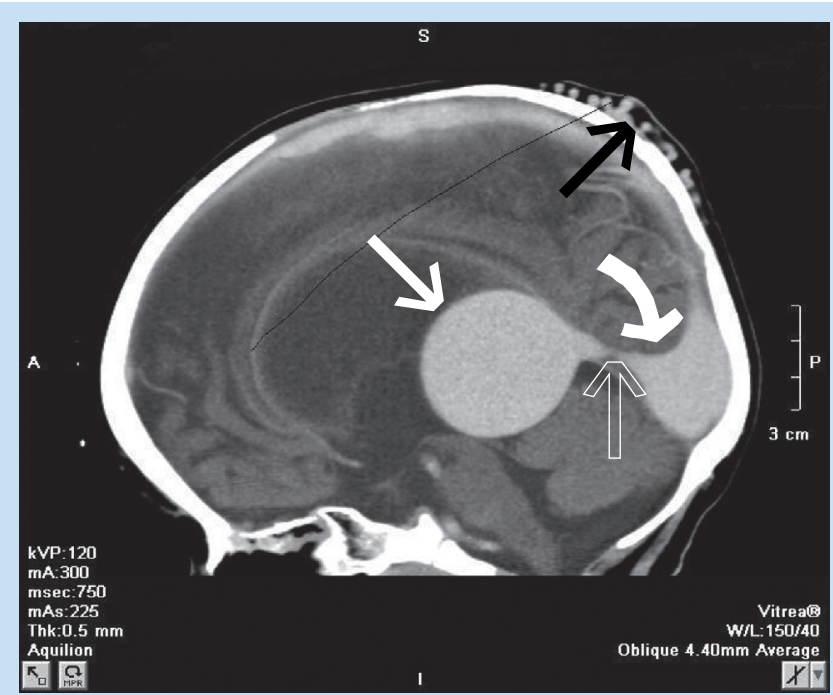

Fig. 1a. Mid-saggital reconstruction of post-contrast CT demonstrating the dilated median prosencephalic vein of Markowski (white arrow) characteristic of VGAM. There is a stenotic straight sinus (open arrow) and a prominent torcular Herophilli (curved arrow). Note also the congested scalp veins (black arrow). 1b, 3a, 3b.) Digital substraction angiography (DSA) confirmed a mural type of VGAM supplied by the left posterior medial choroidal artery. DSA also demonstrated the classical 'jet phenomenon' as contrast flowed into the VGAM through the left medial posterior choroidal artery (Fig. 2).

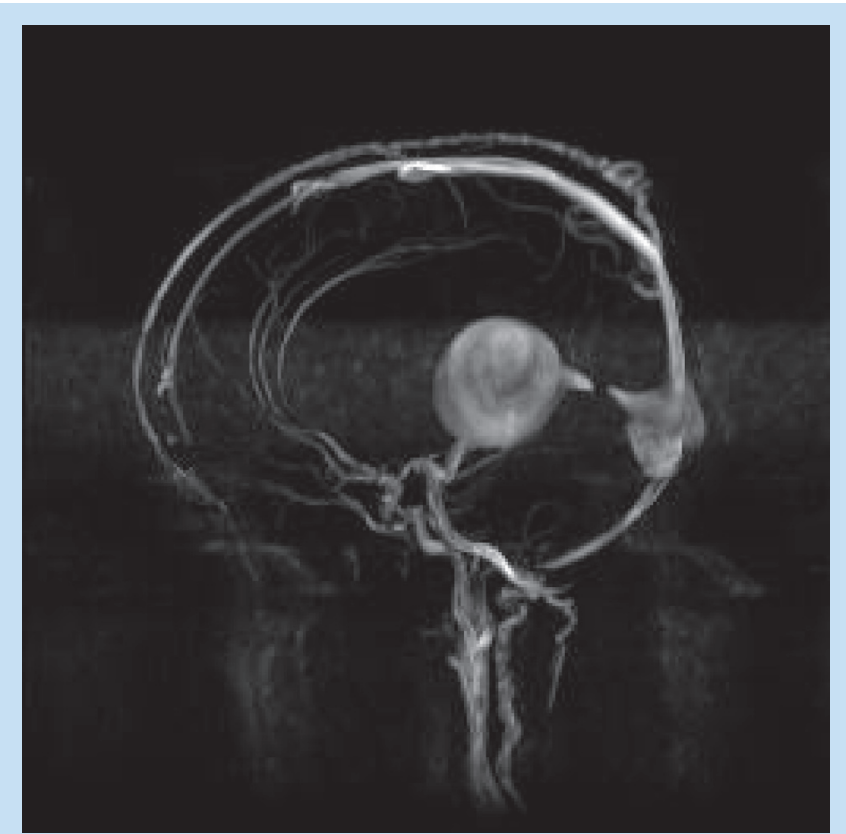

Fig. 1b. Three-dimensional MRV confirming the findings of the postcontrast CT.

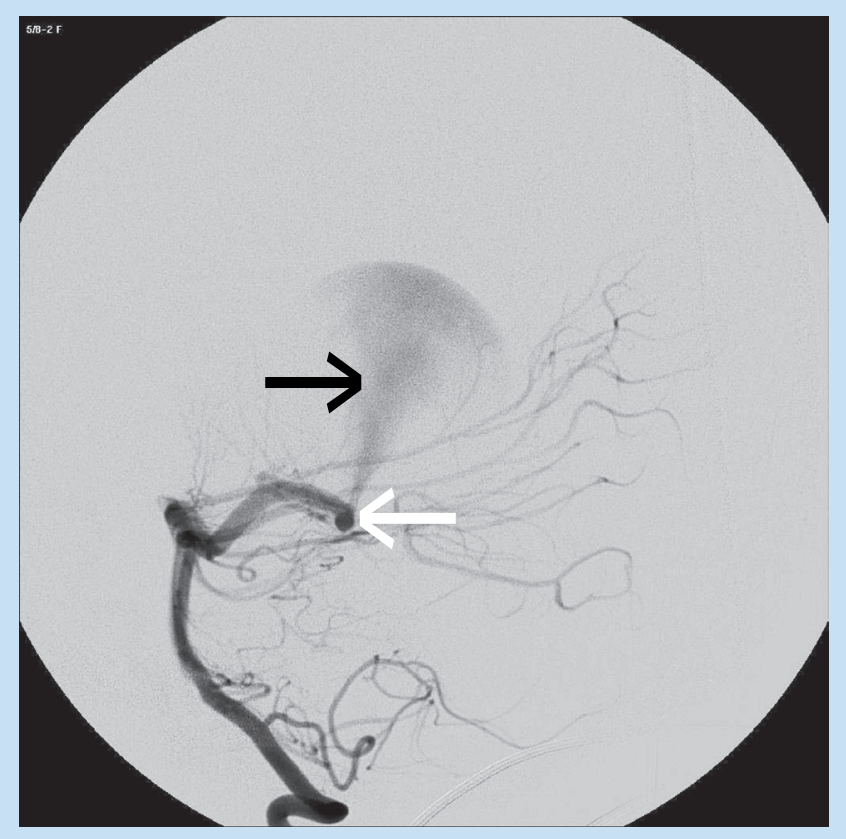

Fig. 2. Lateral vertebral DSA demonstrates the 'jet phenomenon' (black arrow). The VGAM is supplied by the left posterior medial choroidal artery (white arrow). 


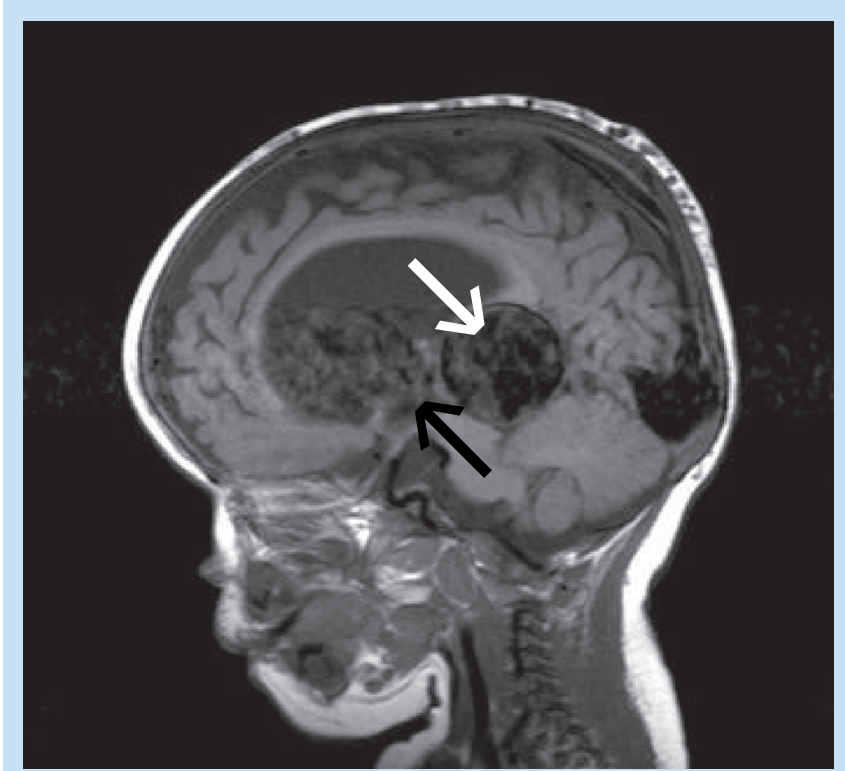

Fig. 3a. On the mid-saggital T1-weighted MRI (pre-contrast) before embolisation, the VGAM is seen as a flow void (white arrow). Note also the flow artifacts (black arrow) at the level of the VGAM.

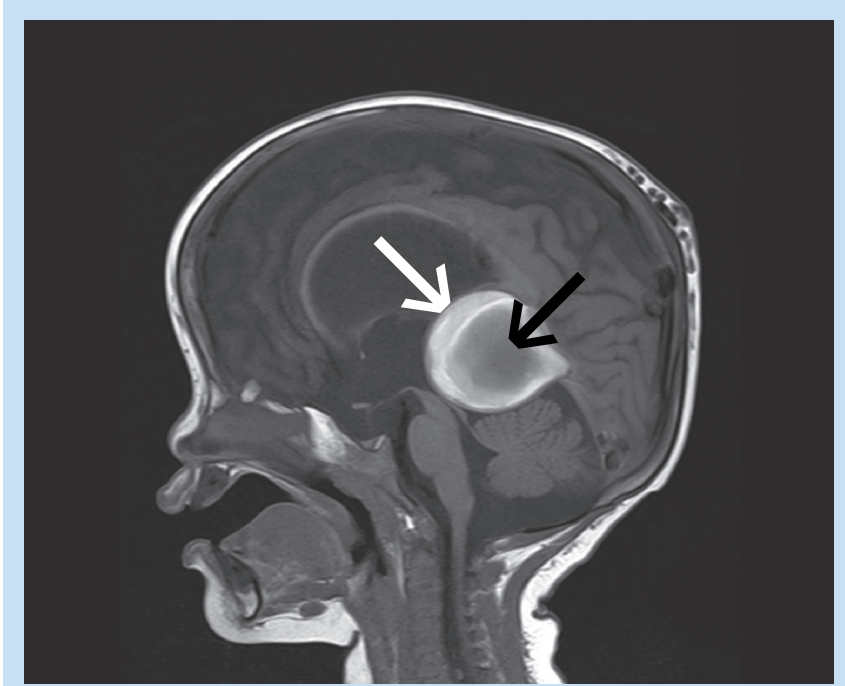

Fig. 3b. Mid-saggital post-embolisation T1-weighted MRI demonstrating thrombosis of the VGAM as evidenced by a peripheral high-intensity (white arrow) and central iso-intensity (black arrow) within the VGAM.

\section{Method}

The foregoing procedures were followed by transarterial embolisation. A micro-catheter was advanced into the posterior medial choroidal artery approximately $1.5 \mathrm{~cm}$ from the fistula leading to the VGAM. A solution of $96 \%$ histo-acryl glue in Lipiodol with tantalum powder was injected through the micro-catheter.

There was very good obliteration of the fistula, with the subsequent control runs showing only small vessels still supplying the aneurysm from the more proximal part of the posterior medial choriodal artery. These smaller vessels were then embolised using a $20 \%$ solution of hysto-acryl glue in Lipiodol. No further significant filling of the aneurysm was seen. A follow-up MRI was obtained (Fig.4.)

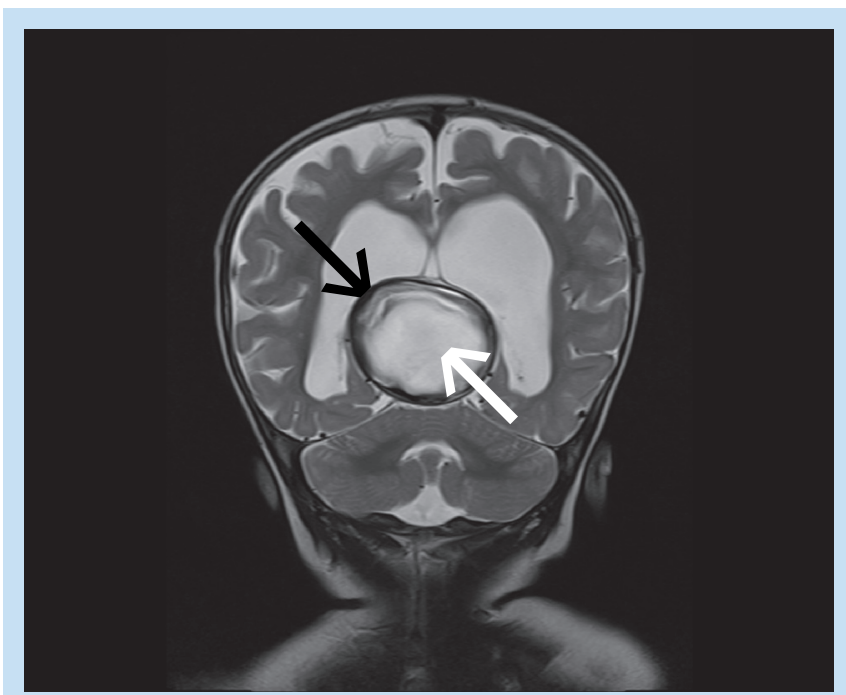

Fig. 4. The VGAM thrombus (white arrow) is seen on coronal postembolisation T2-weighted MRI as a central high-signal intensity (due to methaemoglobin) and a low-signal intensity rim (black arrow) of haemosiderin.

\section{Discussion}

VGAM is divided into choroidal and mural types, based on the arterial supply. ${ }^{1,3,4}$ The choroidal type is supplied by all the choroidal arteries with an interposed network opening into a midline venous pouch. ${ }^{5}$ The arterial feeders are usually bilateral in the choroidal type, and this type is associated with cardiac failure in the neonate. ${ }^{1,4-6}$ In the mural type, direct AV fistulae open on the wall of the MPM, as seen in our case. ${ }^{3-5}$ VGAMs drain via the straight sinus to the torcula but, in the absence of the sinus, drainage is via a persistent falcine sinus to the post third of the superior sagittal sinus. ${ }^{3,5}$ Although this pathway has been challenged by several authors, ${ }^{3}$ the deep venous system is said not to communicate with the VGAM. ${ }^{2,5}$

Associated venous anomalies include anomalous dural sinuses and sinus stenoses. It is postulated that stenosis is the response of the venous endothelium to the rapid and turbulent flow caused by the arteriovenous shunt. ${ }^{3}$ Stenosis of the outflow may beneficially reduce cardiac venous return, preventing congestive cardiac failure. It may, however, also contribute to enlargement of the vein of Galen, with consequent aqueductal compression. ${ }^{6}$

VGAM must be distinguished from vein of Galen aneurysmal dilatation (VGAD). A dural fistula or AVM draining into a true vein of Galen is characteristic of VGAD. ${ }^{2}$ These lesions may present with haemorrhage. ${ }^{4}$

Presentation of VGAM depends on the age of the patient. Neonates, unlike older infants and children, often present with high-output cardiac failure. ${ }^{1,3-5}$ High-output cardiac failure is the result of a steep increase in the cardiac preload owing to the steal phenomenon. ${ }^{7}$ When presenting in older infants, it is usually milder and more responsive to treatment. Hydrocephalus, megalencephaly, developmental delay and seizures are common presentations in older infants and children. ${ }^{1,2,5}$ Hydrocephalus can result from direct aqueductal compression, increased production of CSF owing to increased choroidal blood flow or reduced absorption of CSF because of venous hypertension. ${ }^{6}$ Cerebral hypoperfusion secondary to the steal phenomenon may result in brain infarcts and 
leucomalacia. ${ }^{7,8}$ Congenital cardiac abnormalities may also be present. $^{2}$ Spontaneous thrombosis of VGAM is a rare phenomenon. ${ }^{5}$ When children are referred late, they commonly present with seizures and mental retardation. Imaging may reveal calcification and subependymal, cortical or subcortical atrophy. ${ }^{5}$

After 30 weeks' gestation, VGAM can be detected sonographically as a midline supratentorial cystic mass posterior to the third ventricle demonstrating high-velocity turbulent flow on colour Doppler. ${ }^{8}$ Progressive cardiac dysfunction indicates a high flow lesion that may be unresponsive to treatment. ${ }^{3}$ Fetal MRI can be performed to confirm the antenatal diagnosis. MRI and MRA can aid in diagnosis, as well as in identifying the major arterial supply and venous anatomy. MRI plays an important role in evaluating the brain parenchyma and ventricles, especially after intervention. ${ }^{4}$ Computed tomography (CT) is generally used for screening and evaluating the VGAM. CT angiography can aid in planning embolisation.

Before endovascular treatment, the infant must be evaluated for the development of macrocrania. If there is evidence of preclinical MRI intraventricular hyper-pressure or the head circumference is increasing too rapidly, or there is significant developmental delay, urgent embolisation should be carried out. ${ }^{5}$ Shunting of hydrocephalus before endovascular treatment is generally accepted as the worst option. ${ }^{3,4}$ Shunting is associated with haemorrhage from the dilated superficial veins in VGAM. Progressive hydrocephalus in the post-embolisation period may nonetheless necessitate shunting. ${ }^{3}$

It is generally accepted that a multidisciplinary team approach should be used to manage this condition. ${ }^{4}$ Aggressive medical treatment is needed in the neonate presenting with congestive cardiac failure (CCF). If the CCF continues to worsen, evaluation of the brain, heart, kidney and liver is crucial to determine if endovascular treatment can be offered. ${ }^{9}$ Transarterial embolisation with a liquid acrylic polmer, $n$-butylcyanoacrylate (or 'glue') is the treatment of choice. ${ }^{5}$ A staged approach is advisable. In experienced hands, embolisation can be car- ried out rapidly and the degree of devascularisation can be controlled. ${ }^{2,3}$ Transvenous treatment by packing the venous pouch with coils, balloons and nylon has also been described. ${ }^{5}$ Access for venous occlusion is usually obtained via the femoral or jugular veins, although a transtorcular approach can also be used. Transvenous embolisation is technically less demanding but is reserved for situations where the transarterial method is not feasible. ${ }^{2}$ The venous method has an increased risk of venous infarction and haemorrhage. ${ }^{5}$ The transarterial approach gives a more lasting reversal of CCF compared with the venous approach. ${ }^{9}$

\section{Conclusion}

VGAM is associated with a high morbidity and mortality but, with prompt recognition and appropriate treatment, a good clinical outcome can be achieved.

\section{Acknowledgement}

We thank Professor P A Fourie and Dr P A Scheepers for the transarterial embolisation.

1. Meyers PM, Halbach VV, Phatouros CP, et al. Hemorrhagic complications in vein of Galen malformation. Ann Neurol 2000; 47(6): 748-755.

2. Gailloud P, O'Raidan DP, Burger I, Lehmann CU. Confirmation of communication between deep venous drainage and the vein of Galen after treatment of a vein of Galen aneurysmal malformation in an infant presenting with severe pulmonary hypertension. Am J Neuroradiol 2006; 27: 317-320.

3. Jones BV, Ball WS, Tomsick TA, Millard J, Crone KR. Vein of Galen aneurysmal malformation: Diagnosis and treatment of 13 children with extended clinical follow-up. Am J Neuroradiol 2002; 23: 1717-1724.

4. Mitchell PJ, Rosenfeld JV, Dargaville P, et al. Endovascular management of vein of Galen aneurysmal malformations presenting in neonatal period. Am J Neuroradiol 2001; 22: 1403-1409.

5. Lasjaunias PL, Chng SM, Sachet M, Alvarez H, Rodesch G, Garcia-Monaco R. The management of vein of Galen aneurysmal malformations. Neurosurgery 2006; 59(5): 184-194.

6. Tomsick TA, Ernst RJ, Tew JM, Brott TG, Breneman JC. Adult choroidal vein of Galen malformation. Am J Neuroradiol 1995; 16: 861-865.

7. Lee TH, Shih JC, Peng SSF, Lee CN, Shyu MK, Hsieh FJ. Prenatal depiction of angioarchitecture of an aneurysm of the vein of Galen with three-dimensional color power angiography. Ultrasound Obstet Gynecol 2000; $15: 337-340$.

8. White BD, Kostaki GB, Cacciarelli AA, Madrazo BL. Ultrasound case of the day. Radiographics 1992; 12(2): $396-400$.

9. Terbrugge KG. Vein of Galen management in neonatal period. Am J Neuroradiol 2001; 22: 1236. 